\title{
Missed treatment opportunities and barriers to comprehensive treatment for sexual violence survivors in Kenya: a mixed methods study
}

Anne Gatuguta ${ }^{1,2^{*}}$ (D), Katherine G. Merrill ${ }^{3}$, Manuela Colombini', Seyi Soremekun ${ }^{4}$, Janet Seeley ${ }^{1}$, Isaac Mwanzo ${ }^{2}$ and Karen Devries ${ }^{1}$

\begin{abstract}
Background: In Kenya, most sexual violence survivors either do not access healthcare, access healthcare late or do not complete treatment. To design interventions that ensure optimal healthcare for survivors, it is important to understand the characteristics of those who do and do not access healthcare. In this paper, we aim to: compare the characteristics of survivors who present for healthcare to those of survivors reporting violence on national surveys; understand the healthcare services provided to survivors; and, identify barriers to treatment.

Methods: A mixed methods approach was used. Hospital records for survivors from two referral hospitals were compared with national-level data from the Kenya Demographic and Health Survey 2014, and the Violence Against Children Survey 2010. Descriptive summaries were calculated and differences in characteristics of the survivors assessed using chi-square tests. Qualitative data from six in-depth interviews with healthcare providers were analysed thematically.

Results: Among the 543 hospital respondents, $93.2 \%$ were female; $69.5 \%$ single; $71.9 \%$ knew the perpetrator; and 69.2\% were children below 18 years. Compared to respondents disclosing sexual violence in nationally representative datasets, those who presented at hospital were less likely to be partnered, male, or assaulted by an intimate partner. Data suggest missed opportunities for treatment among those who did present to hospital: HIV PEP and other STI prophylaxis was not given to 30 and $16 \%$ of survivors respectively; $43 \%$ of eligible women did not receive emergency contraceptive; and, laboratory results were missing in more than $40 \%$ of the records. Those aged 18 years or below and those assaulted by known perpetrators were more likely to miss being put on HIV PEP. Qualitative data highlighted challenges in accessing and providing healthcare that included stigma, lack of staff training, missing equipment and poor coordination of services.

Conclusions: Nationally, survivors at higher risk of not accessing healthcare include older survivors; partnered or ever partnered survivors; survivors experiencing sexual violence from intimate partners; children experiencing violence in schools; and men. Interventions at the community level should target survivors who are unlikely to access healthcare and address barriers to early access to care. Staff training and specific clinical guidelines/protocols for treating children are urgently needed.
\end{abstract}

Keywords: Sexual violence, Healthcare, HIV PEP-Kenya

\footnotetext{
* Correspondence: anngatuguta@gmail.com

'Department of Global Health and Development, Faculty of Public Health

and Policy, London School of Hygiene and Tropical Medicine, Keppel Street,

London WC1E 7HT, UK

${ }^{2}$ Department of Community Health, School of Public Health, Kenyatta

University, Nairobi, Kenya

Full list of author information is available at the end of the article
}

(c) The Author(s). 2018 Open Access This article is distributed under the terms of the Creative Commons Attribution 4.0 International License (http://creativecommons.org/licenses/by/4.0/), which permits unrestricted use, distribution, and reproduction in any medium, provided you give appropriate credit to the original author(s) and the source, provide a link to the Creative Commons license, and indicate if changes were made. The Creative Commons Public Domain Dedication waiver (http://creativecommons.org/publicdomain/zero/1.0/) applies to the data made available in this article, unless otherwise stated. 


\section{Background}

Sexual violence is a serious global health problem with significant physical, psychological and social consequences [1-4]. The World Health Organisation (WHO) recommends that survivors of sexual violence should get immediate comprehensive treatment and be followed up for up to 6 months [5]. Immediate treatment involves attention to physical injuries, psychological trauma, prevention of unwanted pregnancy and prevention of HIV and other sexually transmitted infections (STIs) [3, 5]. Long term, survivors of sexual violence are at an increased risk of STIs, abortion, anxiety, depression, post-traumatic stress disorder, suicidal ideation and substance use [2-5]. Furthermore, experiencing sexual violence as a child is associated with negative health outcomes in adulthood including mental health problems, STIs, high risk behaviours such as having multiple sexual partners, unprotected sex, transactional sex and substance use [6-10]. Despite the significant health consequences, research has shown that the majority of survivors do not access healthcare $[2-4,11]$ and many of those who do, do not complete treatment [12-15].

In Kenya, sexual violence is one of the top 10 risk factors for disease burden [16]. National-level data show that $14 \%$ of women and $6 \%$ of men age $15-49$ years have experienced sexual violence in their lifetime [17]. Reported national prevalence is even higher among children and young adults, with $32 \%$ of females and $18 \%$ of males reporting having experienced some form of sexual violence before the age of 18 years [18]. More than $90 \%$ of these survivors do not seek healthcare [17, 18]. Among those survivors who seek healthcare, nearly half do not complete the recommended treatment and follow up $[15,19,20]$. Additionally some survivors present to hospital but are not started on some of the recommended treatment [20].

There is limited data on the reasons why so many survivors in Kenya do not access or complete treatment and why some are not started on treatment even after presenting in hospital. A few studies indicate that factors such as limited financial and human resources; lack of training on managing sexual violence; poor coordination of services; poor referral systems; costs to survivors; stigma; and, lack of active follow may contribute $[15,19-21]$. Population-level data also suggest that individual characteristics such as age, marital status, place of residence, employment status, level of education and whether one has experienced both physical and sexual violence may influence help-seeking to deal with the sexual violence from either formal or informal sources [17].

In order to appropriately target interventions to improve healthcare seeking and treatment completion, a better understanding of why many survivors do not obtain or complete treatment is required. Little is known about characteristics of survivors who are least likely to access healthcare in Kenya. Healthcare providers' perspectives are important in understanding the gaps in care for survivors who do present for healthcare. This study therefore aimed to: 1) compare the characteristics of survivors who present for healthcare at two hospitals to those of survivors reporting violence on national surveys; and 2) understand the services provided to survivors who present for healthcare and identify barriers to treatment.

\section{Methods}

\section{Setting}

Kenya has a six-tiered (level 1-6) healthcare system, with lower level tiers offering basic healthcare services and more specialised services available in higher-level facilities. From September to November 2015, a review of survivor data and in-depth interviews were conducted in a level four hospital (Naivasha Sub-county Referral Hospital) and a level five hospital (Thika Level 5 Hospital). Naivasha Sub-county Hospital is situated within the Nakuru County approximately $90 \mathrm{~km}$ northwest of the capital city Nairobi and, Thika Level 5 Hospital is located within the Kiambu County, which borders the capital Nairobi to the east. Both facilities ran an outpatient service that attends to walk-in patients, including sexual violence survivors. The two facilities were purposively selected as they both treat a high number of sexual violence survivors. Additionally, these facilities are presumably fully equipped to offer all the services required by survivors: they have trained medical personnel, all the medicines including HIV PEP, equipped laboratories, required documents and trained counsellors. Lower-level facilities often lack one or more components of the services and therefore, these two hospitals also receive survivors referred from the lower levels.

\section{Design}

A mixed methods approach was used. Quantitative hospital data were collected through existing hospital records and qualitative data through in-depth interviews with healthcare providers. The hospital data aimed to establish the characteristics of sexual violence survivors presenting to the facility, the treatment provided and challenges encountered by healthcare providers in the process of treating survivors. Subsequently, the hospital data were compared with population-level data from national surveys to establish if any differences existed between the profile of those survivors 
reporting violence nationally and those accessing care in the hospitals.

\section{Quantitative data Hospital data}

Data were abstracted from post-rape care (PRC) forms into a standardised excel sheet. The PRC form is filled in for every survivor presenting at the hospital and is used for both medical and legal purposes. It documents the socio-demographic characteristics of the survivor, the nature of the sexual violence act, where it occurred and when, who the perpetrator was, the physical examination findings on presentation, investigations done, results of the investigations, treatment given and referral to any other services [22]. A total of 543 survivor records were abstracted. Once data were abstracted, all the entries were double-checked against each PRC form filling in any missing values. Where missing values or outliers could not be resolved from the PRC form only, other sources such as the counsellor's records and the sexual and gender-based violence (SGBV) register (contains summarised data for all survivors seen at the hospital) were used. Other missing data were extrapolated judiciously. For example, if gender was missing but there was a record of a pregnancy test or contraceptive being issued, female gender was assigned.

\section{Survey data}

National data were obtained from the Kenya Demographic and Health Survey (KDHS) 2014 and Violence Against Children Survey (VACS) 2010 [17, 18]. The KDHS 2014 survey interviewed both men and women 15-49 years on their lifetime and current experience of sexual violence and help seeking. The VACS 2010 is a cross- sectional household survey of 13 to 24 year old females and males designed to produce national-level estimates of the prevalence of violence against children. The survey data methods for KDHS 2014 and VACS 2010 are described fully in the KDHS 2014 and VACS 2010 reports respectively $[17,18]$.

\section{Measures (Appendix 1)}

Respondents were classified as having experienced sexual violence if reporting having ever been a victim of one or more behavioural acts of sexual violence (e.g. physically forced to have sex against will, pressured to have sex when sex was unwanted, etc.). Binary variables were used to assess respondents' sex, whether the respondent knew the perpetrator of sexual violence, and whether or not the perpetrator was a current/previous partner. We considered age as a categorical variable. We grouped marital status according to whether the respondent was: currently married or cohabitating; previously married or cohabitating; or single. Finally, we generated a variable with six groupings to consider the place where the violence occurred: at the respondent's own home; the perpetrator's home; someone else's home; while traveling by foot or roadside or bush; school; or at another location (e.g. a party, public event, etc.).

\section{Statistical analysis}

Analyses were performed using STATA 14. Participants with missing data were omitted from the analyses where the missing values were present. To compare the characteristics of survivors who present for healthcare at the two hospitals to those of survivors reporting violence on national surveys, we appended the following datasets: the male and female datasets from the KDHS 2014, the male and female datasets from the VACS 2010, and our hospital dataset. We restricted our analysis to participants aged 15 years and above who reported having ever experienced sexual violence $(n=353$ hospital respondents, $n=1069$ KDHS respondents, $n=611$ VACS respondents). Descriptive statistics were computed and characteristics of hospital survivors, those reporting sexual violence in the KDHS and the VACS were compared using chi-square or Fisher's exact tests as appropriate.

\section{Qualitative data}

In-depth interviews were conducted with two trauma counsellors, two clinical officers and two nurses were selected. These healthcare professionals provided services directly to the survivors, interacted most with survivors at each of the facilities, and were involved in their follow up. At each hospital, participants were identified with the help of a nurse counsellor who is the hospital's SGBV coordinator. Eligible participants were doctors, clinical officers (COs), nurses and counsellors. Both male and female healthcare providers were interviewed. Topic guides were used to explore healthcare providers' views on characteristics of survivors; barriers to survivors seeking and completing care; and, challenges of providing services to survivors.

\section{Interview procedures}

AG conducted all the interviews in a private room within the hospital. All interviews were conducted in English. Written informed consent was obtained from all participants. All interviews were audio-recorded with permission from interviewees and lasted for about 30-60 $\mathrm{min}$.

\section{Data analysis}

Interviews were transcribed verbatim and checked against recordings. The transcripts were then imported to NVivo 11 software. Data were analysed thematically 
[23]. Initial themes and codes were developed by AG based on study objectives and a literature, and were reviewed by KD. During coding, any emerging new ideas and concepts from the data not categorised previously were given new themes and codes. Once data were coded, the materials with similar codes were sorted and grouped together through thematic matrices with categories and sub-categories under the main themes.

\section{Ethical approval}

Ethical approval was obtained prior to commencement of the study from the institutional ethics review committees of the London School of Hygiene and Tropical Medicine (Ref. 9896) and Kenyatta University (Ref. PKU/386/E32).

\section{Results}

Socio-demographic characteristics of survivors presenting for treatment (hospital respondents)

The socio-demographic characteristics of survivors presenting at the two hospitals are summarised in Appendix 2. The majority, 506 out of 543 (93.2\%) were female. The mean age was 16.8 years, with the majority (69.5\%) being children below 18 years (range 1-67 years). Among adult survivors whose marital status was documented $(n=177)$, the majority were single 123 (69.5\%), followed by married $32(18.1 \%)$, separated $8(4.5 \%)$, widowed $7(3.9 \%)$ and divorced 7 (3.9\%).

In majority of the cases, 461 out of 530 (87\%), only one perpetrator was reported. The incidence occurred in the perpetrator's home in 183 out of 407 (45\%) of the times, followed by the roadside or bush 83 (20.4\%), and in survivor's own home 71 (17.4\%). Most, 387 out of 538 (71.9\%), of the survivors knew the perpetrators. Neighbours $30.6 \%$, friends $17.3 \%$ and relatives $16 \%$ were among the most common known perpetrators of violence. Cases of adolescent girls willingly engaging in sex with their boyfriends and sometimes leaving home to cohabit with them accounted for a significant proportion (15.3\%) of those treated. As these girls had not reached the legal age of consent, parents brought them to the hospital as cases of statutory rape.

The age and gender of respondents in the two hospitals were similar but survivors differed in marital status, type of perpetrator, number of perpetrators and place of violence (Appendix 2).

\section{Socio-demographic characteristics of hospital respondents compared to survivors reporting violence in KDHS 2014 and VACS 2010 \\ Gender}

The proportion of men reporting violence nationally differs significantly to those seeking healthcare at the hospitals (Table 1 ). While only $4 \%$ of those presenting for treatment are men, $28 \%$ of the KDHS respondents and $39 \%$ of VACS respondents reporting sexual violence are men $(p<0.001)$. This translates to only one man for more than twenty women among survivors who seek healthcare, compared to nearly one man for every three women reporting violence nationally.

\section{Age}

The age profile of survivors reporting violence in the hospital data also contrasts sharply with national reporting of violence, suggesting that older survivors are less likely to seek healthcare compared to younger survivors. While the hospital data indicate that children 1519 years constitute the highest proportion (57\%) of survivors presenting for care, KDHS 2014 data show that adult survivors are more likely to report experience of violence $(p<0.001)$. For instance, only $6.5 \%$ of those reporting violence are 15-19 years with the highest proportion of survivors being 25-30 years (23\%), followed by $30-34$ years (18\%) and 35-39 years (16\%). Similarly, while more than $20 \%$ of women who reported violence in the KDHS 2014 are 40 years and above, less than $5 \%$ of those presenting for healthcare fall in this age group.

\section{Marital status}

Among the hospital respondents, never married survivors were more likely to present for treatment while currently married/cohabiting survivors were more likely to report experience of violence nationally $(p<0.001)$. Among the KDHS respondents, the proportion of never married survivors was $11 \%$, currently married/ cohabiting $70 \%$ and previously married/cohabiting $18 \%$. In contrast, survivors presenting at hospital were overwhelmingly single (84\%), compared to currently married/cohabiting (10\%) and previously married/cohabiting (7\%).

\section{Perpetrators of violence}

There were differences in the type of perpetrators reported by the national surveys respondents and hospital respondents. Hospital respondents were more likely to be assaulted by strangers $(66 \%)$ compared to VACS respondents $(54 \%)(p<0.001)$. Current and past intimate partners were the most commonly reported perpetrators of violence among VACS and KDHS respondents but represented less than 19\% of perpetrators among hospital respondents $(p<0.001)$. Notably, among hospital respondents reporting violence from intimate partners, a majority $(76 \%)$ were adolescents who reported the sexual encounter as consensual but were 
Table 1 Characteristics of participants ages 15+ reporting having ever experienced sexual violence across hospital, Violence Against Children, and Kenya Demographic and Health surveys

\begin{tabular}{|c|c|c|c|c|c|}
\hline & & $\begin{array}{l}\text { Hospital } \\
\text { Respondents }\end{array}$ & $\begin{array}{l}2010 \text { VACS } \\
\text { Respondents }\end{array}$ & $\begin{array}{l}2014 \mathrm{KDHS} \\
\text { Respondents }\end{array}$ & $p$ value \\
\hline$\overline{\text { Total }^{a}}$ & & $353(100 \%)$ & $611(29.0 \%)$ & $1069(10.1 \%)$ & \\
\hline \multirow[t]{2}{*}{ Sex } & Male & $15(4.3 \%)$ & $240(39.3 \%)$ & $301(28.2 \%)$ & \multirow[t]{2}{*}{$<0.001$} \\
\hline & Female & $338(95.8 \%)$ & $371(60.7 \%)$ & $768(71.8 \%)$ & \\
\hline \multirow{8}{*}{$\begin{array}{l}\text { Age }(n=297 \text { hospital } \\
\text { respondents) }\end{array}$} & $15-19$ & $169(56.9 \%)$ & $272(44.5 \%)$ & $69(6.5 \%)$ & \multirow[t]{8}{*}{$<0.001^{*}$} \\
\hline & $20-24$ & $49(16.5 \%)$ & $339(55.5 \%)$ & $163(15.3 \%)$ & \\
\hline & $25-29$ & $39(13.1 \%)$ & $/ /$ & $250(23.4 \%)$ & \\
\hline & $30-34$ & $20(6.7 \%)$ & $/ /$ & $194(18.2 \%)$ & \\
\hline & $35-39$ & $6(2.1 \%)$ & $/ /$ & $172(16.1 \%)$ & \\
\hline & $40-44$ & $6(2.1 \%)$ & // & $122(11.4 \%)$ & \\
\hline & $45-49$ & $3(1.0 \%)$ & $/ /$ & $81(7.6 \%)$ & \\
\hline & $50+$ & $5(1.7 \%)$ & // & $18(1.7 \%)$ & \\
\hline \multirow{3}{*}{$\begin{array}{l}\text { Marital status( } n=338 \text { hospital } \\
\text { respondents; } n=610 \text { VACS respondents) }\end{array}$} & Currently married/cohabitating & $32(9.5 \%)$ & $184(30.2 \%)$ & $753(70.4 \%)$ & \multirow[t]{3}{*}{$<0.001$} \\
\hline & Previously married/ cohabitating & $22(6.5 \%)$ & $34(5.6 \%)$ & $197(18.4 \%)$ & \\
\hline & Single & $284(84.0 \%)$ & $392(64.3 \%)$ & $119(11.1 \%)$ & \\
\hline \multirow{2}{*}{$\begin{array}{l}\text { Perpetrator known? ( } n=349 \\
\text { hospital respondents) }\end{array}$} & Yes & $119(34.1 \%)$ & $282(46.2 \%)$ & $/ /$ & \multirow[t]{2}{*}{$<0.001^{* *}$} \\
\hline & No & $230(65.9 \%)$ & $329(53.9 \%)$ & $/ /$ & \\
\hline \multirow{2}{*}{$\begin{array}{l}\text { Relationship of perpetrator to survivor } \\
\text { ( } n=291 \text { hospital respondents; } n=289 \\
\text { VACS respondents) }\end{array}$} & Current/previous partner $^{\mathrm{b}}$ & $54(18.6 \%)$ & $147(50.9 \%)$ & $589(55.1 \%)$ & \multirow[t]{2}{*}{$<0.001$} \\
\hline & Other & 237 (81.4\%) & 142 (49.1\%) & 480 (44.9\%) & \\
\hline \multirow{6}{*}{$\begin{array}{l}\text { Place where violence occurred } \\
n=257 \text { hospital respondents; } n=366 \\
\text { VACS respondents) }\end{array}$} & Own home & $41(16.0 \%)$ & 68 (18.6\%) & $/ /$ & \multirow[t]{6}{*}{$<0.001^{* *}$} \\
\hline & Perpetrator's home & $110(42.8 \%)$ & $54(14.8 \%)$ & $/ /$ & \\
\hline & Someone else's home & $12(4.7 \%)$ & $20(5.5 \%)$ & $/ /$ & \\
\hline & Traveling by foot/roadside/bush & $65(25.3 \%)$ & 70 (19.1\%) & $/ /$ & \\
\hline & School & $1(0.4 \%)$ & $77(21.0 \%)$ & $/ /$ & \\
\hline & Other & $28(10.9 \%)$ & $77(21.1 \%)$ & $/ /$ & \\
\hline
\end{tabular}

$p$ values assessed using chi-squared tests

$V A C S$ violence against children survey, KDHS Kenya demographic and health survey

* $p$ value comparing Kenya Demographic and Health Survey respondents with hospital respondents only

${ }^{* *} p$ value comparing Violence Against Children Survey respondents with hospital respondents only

${ }^{a}$ Represents the proportion of respondents for each dataset who report having ever experienced sexual violence

${ }^{b}$ For hospital respondents reporting violence by current/former partner, 41 of 54 (76\%) were children below 18 years and believed that the sexual encounter with the boyfriend/girlfriend was not coerced; the parent, however, reported the encounter as coerced and took them for treatment

brought to the hospital by disapproving parents as cases of statutory rape.

\section{Place of violence}

The place where violence occurred was reported only among hospital respondents and VACS respondents. However, not all of these respondents reported this; these data were missing among 27 and $40 \%$ of hospital and VACS respondents respectively. Among hospital respondents with data, violence occurred most commonly in the perpetrator's house (43\%), followed by while travelling on foot, by the roadside or bush (25\%). Among the VACS responds, school was the most common place of violence $(21 \%)$ followed by travelling on foot, roadside or bush (19.1\%). Although most hospital respondents are of school-going age (15-19 years), it is surprising that violence reported to have occurred in school was very low (less than 1\%) compared to the VACS.

\section{Quantitative findings on services provided to survivors in the hospital and barriers to treatment}

Similar services were offered in the two hospitals. The services comprised of an initial medical history and physical examination; specimen collection and laboratory tests; treatment; and, counselling. The tests done included screening for STIs such as HIV, syphilis and hepatitis B; a pregnancy test; urine and high vaginal swab microscopy; haemoglobin and liver function tests. The treatment recommended includes HIV PEP, emergency contraceptive (EC), STI prophylaxis, anti-tetanus and hepatitis $B$ vaccines as needed. In Naivasha Sub-county Hospital, the services were offered across 
different departments (outpatient, casualty, HIV comprehensive care centre, Youth centre, and Pharmacy) situated in separate buildings, ranging from a few metres to over $100 \mathrm{~m}$ apart. In Thika Level 5 Hospital, all services were provided at the HIV comprehensive care centre (except at night and weekends when services were offered in the outpatient department). The HIV comprehensive care centre comprised of two buildings adjacent to one another with different services offered in separate rooms.

\section{Missing clinical examination findings and laboratory test results}

Missing reports of clinical findings and laboratory tests results were common (Appendix 3). It was not clear from the records whether the absence of these reports was due to the services not being offered or due to failure to document the services. Physical examination findings were not documented in 5\% of the survivors, psychological assessment in $14 \%$ and genital exam in $2 \%$. In some cases, examination findings documented were very broad and/or uninformative and therefore unlikely to be useful in evaluating a patient clinically or as forensic evidence. For instance, in $46 \%$ of survivors, genital exam was reported only as "hymen broken" with no details to indicate whether this was a fresh injury or not and whether there were any other relevant genital findings suggestive of sexual violence. Failure to carry out laboratory investigations and/or to document results was particularly prominent. None of the laboratory tests had complete records: $40 \%$ HIV, $42.2 \%$ urinalysis, $40.3 \%$ swab microscopy, $43.3 \%$ VDRL and 50.4\% Hepatitis B had no results.

\section{HIV PEP, other STI prophylaxis and emergency contraception}

Review of records indicated that some survivors presented to the hospitals but did not obtain certain components of the recommended treatment (Table 2). According to the national treatment guidelines, all survivors presenting within $72 \mathrm{~h}$ of assault and who have a significant risk of HIV exposure (such as those with oral, vaginal and anal penetration) are eligible for HIV PEP. Among all survivors presenting at the two hospitals, $30 \%$ did not receive HIV PEP. The likelihood of not being put on HIV PEP was associated with the age of survivor $(p<0.001)$, whether the perpetrator was known or unknown $(p<0.001)$ and the relationship of the survivor to the perpetrator $(p=0.002)$. Children aged 18 years and below were more likely to miss being put on HIV PEP (37\%) compared to survivors above 18 years $(14 \%)$. Survivors where the perpetrator was known were also more likely to miss being put on HIV PEP (38\%) compared to survivors where the perpetrator was unknown (11\%). Among those who were not put on HIV PEP, the most common reason for not being put on HIV PEP was late presentation (59\%). Among children 18 years or below, $67 \%$ of those who did not get HIV PEP presented late. Survivors where the perpetrator was known were also more likely to miss HIV PEP because of late presentation (62\%) compared to survivors where the perpetrator was unknown (27\%). Notably, $24 \%$ of all survivors who did not get HIV PEP presented within $72 \mathrm{~h}$ and no reasons were documented for not starting them on HIV PEP.

Prophylaxis for other sexually transmitted infections was given in $84 \%$ of all survivors. Other than whether a perpetrator was known or unknown $(p=0.002)$, no other survivor characteristics were associated with being put on STI prophylaxis. Among women whose records documented whether EC was given or not, 57\% received EC. Single women were more likely to receive EC $(p=0.027)$ compared to previously partnered or married women. Similarly, women where the perpetrator was unknown were more likely to receive EC compared to women where the perpetrator was known $(p=0.001)$.

\section{Qualitative findings on socio-demographic characteristics of survivors}

Healthcare providers' (HCPs) views expressed during the interviews helped to explain some of the observed differences between survivors presenting at the hospitals and those reporting violence nationally.

\section{Stigma influences the type of survivors seeking healthcare}

Sexual violence stigma influenced the type of survivors presenting at the hospitals. According to HCPs, men were under-represented among the survivors treated compared to the numbers in the community as men were afraid of identifying as survivors at the community level. The HCPs observed that stigma at the community level also affected adult females. A culture of blaming women for provoking the violence through dressing skimpily, being in the wrong place or through their work prevented them from disclosing and seeking treatment.

"They [men survivors] are there but they do not come. Because, there is that stigmatisation and that fear of how the community is going to portray you." (Professional healthcare provider, HCP3)

"Ok some people relate it [violence] with your job. Probably you are a bar maid, so even if you are raped they usually take it that you are the one who 


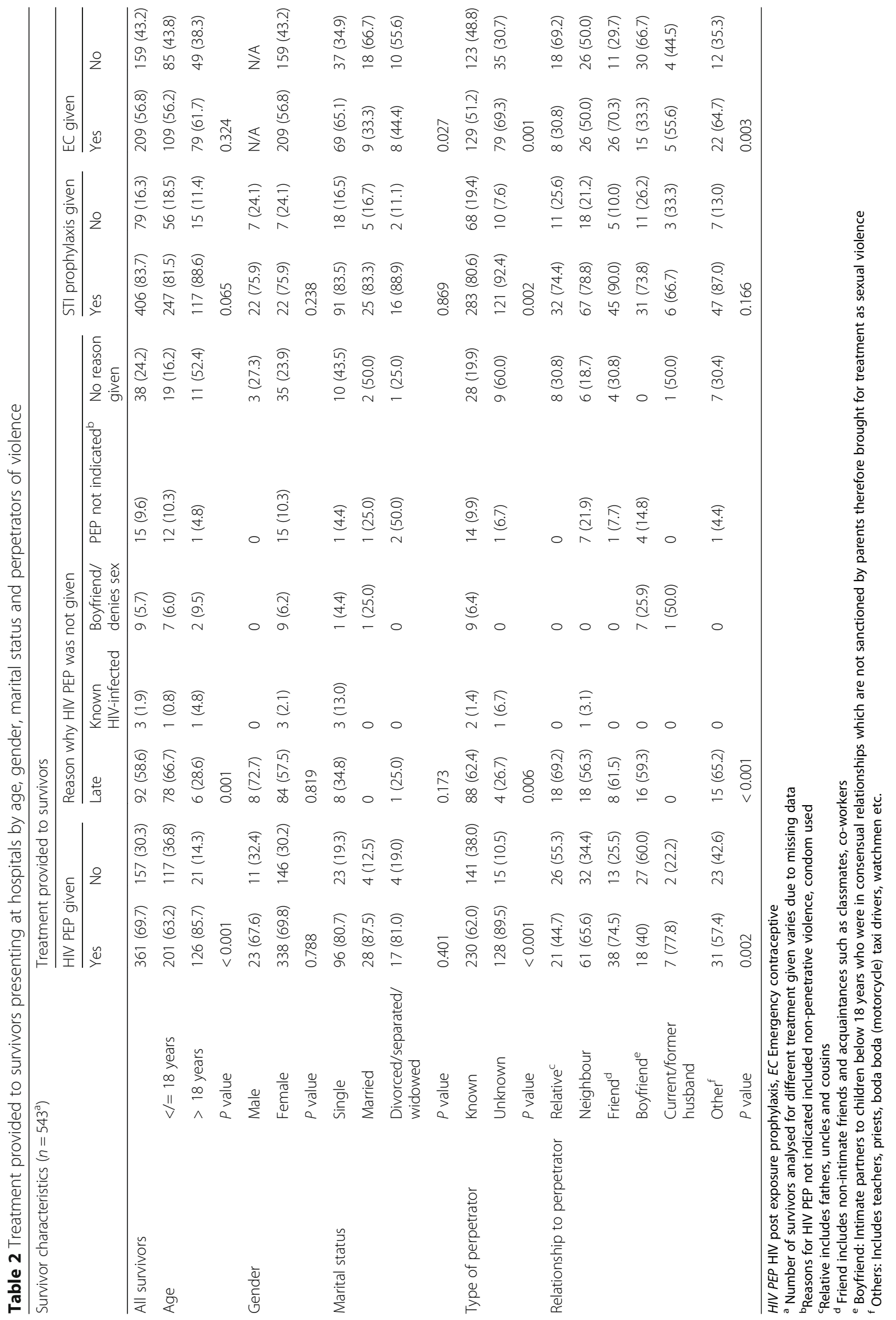


provoked the perpetrator. And then again, also some in the community, how you dress. They tell you now you are the one who attracted the perpetrator to you." (Professional healthcare provider, HCP5)

Healthcare providers reported that most of the survivors were from disadvantaged and poor backgrounds. They were also more likely to come from particular communities within the hospital's catchment area. The fact that survivors were more likely to come from certain areas could have implications on sexual violence prevention. Cultural norms and the status of women in society were reported as predisposing factors to sexual violence in these areas. Survivors from such areas were likely to present for treatment only after multiple incidents of violence and many other cases in such areas were unreported.

"Let me say the community that lives there [an area where many of the survivors come from], the men, they don't value so much a woman according to what they tell me. They consider a young person, a young woman, a young girl as a wife. So anytime there is a case that happens, when they come, they report that it's something that has been going on for a while (...). The other reason is they (the women) are also not learned. They don't have the knowledge of their sexual and reproductive health rights." (Professional healthcare provider, $\mathrm{HCP} 3$ )

HCPs therefore highlighted the importance of community awareness in improving healthcare seeking and follow-up. There was reported lack of general awareness on sexual violence in the community and lack of awareness of available services and where to find them. The lack of awareness is also compounded by the level of stigma in certain communities. HCPs observed that awareness creation would enable survivors to present for care by reducing community stigma and also being aware of the available services and the importance of seeking and completing treatment.

"The awareness needs to be raised so that people don't feel that it's awkward to go to the hospital when you're sexually assaulted. Because, in as much as I've said it's not there [stigma], there is still some component of it in the village, especially in the village because those who come directly, most of them are from the town [...] So, campaigns to raise awareness through the community health workers, something of the sort." (Professional healthcare provider, $\mathrm{HCP} 1$ )

\section{Types of perpetrators}

The profile of perpetrators as reported by healthcare providers mirrored that observed among hospital respondents' quantitative data. Interviewed healthcare providers elaborated on the kind of perpetrators reported and the circumstances in which violence took place. Reported perpetrators were mostly well known particularly in children and adolescents. Grooming in young children was reported as the main reason why children did not report violence as the perpetrators enticed children with gifts often building a relationship over time before the actual sexual act. In contrast, adolescents were often taken advantage of in circumstances where they had diminished capacity to make decisions.

"They (perpetrators) are of course people well known especially to the children and even I have realised, even to adults. Occasionally you will get those who do not purely know the survivors, but especially in adolescents, majority of them they know the survivors, they are their friends, they go for parties in company of others, when they are drunk, they are taken advantage of (...). Even in children because when they come, those ones of a young age, they will talk of uncle or even a relative and not uncle in quotes but a real uncle. Others step fathers, occasionally even a father." (Professional healthcare provider, $\mathrm{HCP} 4$ )

Quantitative findings indicated that some adolescents were treated as sexual violence survivors even though they reported being in consensual relationships, sometimes even cohabiting with the alleged perpetrators. During follow up informal discussions to clarify this, HCPs observed that some of the adolescents were brought to hospital by parents/guardians who did not approve of these relationships. As the adolescents had not reached the legal age of consent, they were brought as cases of statutory rape. Parents could then use evidence obtained from hospital to prosecute the alleged perpetrator and stop the relationship.

Qualitative findings on barriers to accessing and receiving quality treatment

Interviewed HCPs provided some insight to the process of getting treatment once at the hospital, reasons as to why some expected treatment might not have been given as well as challenges associated with providing treatment. 


\section{Late presentation}

HCPs reported that survivors gave various reasons for presenting late-the most common reason for not getting HIV PEP — such as distance, cost of transport to get to the hospital, fear of disclosure and threats from the perpetrator. Late presentation was particularly common in children, as children often did not disclose the abuse immediately due to fear and abuse was often discovered after complications set in.

"The main challenge is the children. Because you see most of the times after the ordeal has happened, these children are threatened. They don't talk about it until maybe much later when the parent or the guardian may realise there is a problem, or maybe immediately the guardian may realise am seeing some funny behaviours or I can see there is something wrong with my child and they try to peruse and they will come out with the information. But if not very severely damaged, these children will keep quiet. You know most of the times, they say they are enticed, they are given gifts, they are given biscuits and all that. So they don't really talk about it [...]. So that is why some of these children will come late and they don't get the services as required." (Professional healthcare provider, $\mathrm{HCP} 4$ )

\section{Poor coordination of services}

Facility-related challenges were also identified that hindered efficient treatment of survivors once at the hospital. Survivors being attended to at different service delivery points, in different buildings resulted not only in delays but also survivors being lost along the in-hospital pathways.

Interviewer: Are there situations where they don't get there (counseling department)?

Respondent: "Yes there are situations. Because, the ones who don't go... are the ones who have come and we [clinicians] have seen them, have filled the forms, but have been kept waiting for long hours in the outpatient department. They feel frustrated. And they have that mentality that if I go to the other centres am being sent to, I will still wait the way I have just been waiting in the outpatient department." (Professional healthcare provider, $\mathrm{HCP} 3)$

Despite the fact that there was hospital policy that survivors should be treated as soon as possible without waiting in queues, survivors were still kept waiting for a long time in some service delivery points. Various reasons contributed to survivors waiting and included clinicians being busy with other patients and delays in some departments.

"Timeliness is still an issue. Because, we are really not seeing them with the speed that we would like to see them with. Maybe delays have been caused, maybe the survivor is supposed to see a clinical officer and maybe the clinical officer is attending to another patient [...]. We are also seeing delays sometimes in the lab." (Professional healthcare provider, HCP2)

\section{Lack of equipment}

Lack of necessary equipment to carry out comprehensive management such as speculums and rape kits was also a hindrance to survivor's care. Furthermore, there were occasions when HIV test kits were missing and survivors were forced to buy them. This could perhaps explain the fact that $40 \%$ of survivor records did not have swab and HIV results. The lack of equipment limited the quality of care that could be provided to survivors. Lack of speculums, for instance, limited the ability of clinicians to collect quality specimens necessary for both treatment and forensic evidence.

"Sometimes we lack even the kits [HIV test], like now it's only me who has them. I only have a few, so if they want to test like now this lady who was here [another counsellor] they come for them [...] sometimes we don't have. [...] We tell them [survivors] to do it outside and come with results." (Professional healthcare provider, HCP6)

"Well, challenge with speculums, they aren't enough. There are times you may go for a speculum and you are told right now we don't have. So you'll keep the patient waiting and, some clinician's do it (high vaginal swab) without, which really should not be the case. It's a malpractice because if you do it without a speculum, you are likely to even introduce more infections and the results also can be affected by that." (Professional healthcare provider, HCP2)

\section{Few trained HCPs}

Limited training was noted as a major hindrance to providing quality services to survivors. In two departments at the forefront of survivor's care, HCPs estimated that only about three of approximately 13 staff 
working in the department were trained to handle survivors. Moreover, although children were the most common survivors, none of the HCPs had training on dealing with child survivors. This shortage limited the number of staff members who were confident in managing the survivors, impacting negatively on the treatment of survivors. In some instances, survivors had to wait for a particular individual to receive certain services.

"I think what we can do is, one, we need CMEs (continuous medical education) and workshops. At least all health workers should know what to do to such clients because if we all depend on [name of colleague] that she is the one who has done sexual based violence, she can be able to do $1,2,3 \ldots$ It will be quite hard, because some people- right now, at least I am better off because I went for a workshop once for gender-based violence. But you see some people have not, and you know I also won't be at work throughout." (Professional healthcare provider, $\mathrm{HCP} 5$ )

\section{Lack of follow up}

The hospitals did not have a formal way of following up survivors to ensure that they returned to complete their treatment. Healthcare providers estimated that only about $30-50 \%$ of survivors came back after the initial treatment. The HCPs noted that they were limited in their ability to carry out the role of following up survivors. Instead, this would only be possible through involving other players at the community level such as community health workers.

"As a clinician I think it would only be possible [to follow up] if the community is involved. Because as a clinician I don't think I can be able to follow up anyone unless I have special interests, unless I want to. Because even the other patients the general patients, I don't get to know what happened to them unless I have a special interest." (Professional healthcare provider, $\mathrm{HCP} 1$ )

\section{Discussion}

Three key findings emerged from this study: 1) Certain groups of survivors reporting violence nationally are unequally represented in seeking treatment; 2) within health facilities, various gaps exist leading survivors to miss out on essential treatment even after presenting for care; and, 3) while children below 18 years form a disproportionately large proportion of survivors presenting to hospitals, there are exceptional limitations to children's services.

\section{Discrepancies in survivors reporting violence nationally and those presenting for treatment in hospital}

Although national survey data show that reporting of violence increases with age and older survivors are more likely to report sexual violence [17], our hospital data seems to contradicts this. Together with multiple other studies in Kenya [15, 19-21, 24], our data show that children are more likely to present for treatment in hospitals. Moreover, the proportion of male survivors presenting for care is low compared to the proportion reporting violence, indicating that the vast majority of men who experience sexual violence do not seek healthcare. The reasons why older female survivors and men in Kenya do not access healthcare are not clearly documented, however, our qualitative data from HCPs suggests that stigma is a significant reason. Studies on healthcare seeking for sexual violence, especially in men, in the African setting are limited. Our findings correspond to a study done in the Democratic Republic of Congo that similarly identified stigma and shame as well as other barriers such as costs of care and limited availability of services as contributing factors to men missing out on treatment [25]. It is possible that the higher reporting in older women and men but less treatment seeking may be due to adults reporting incidences of violence that occurred during their childhood. However, the fact that the national survey also shows that most survivors are currently married/cohabiting and the majority are assaulted by current/previous intimate partners- incidences that likely occurred in adulthoodsuggests that the low numbers of adults in hospital are more likely due to failure to seek treatment. More studies in the local context; efforts to identify those affected but not receiving treatment; and, targeted services for men are needed.

The current quantitative data also suggest that survivors experiencing intimate partner sexual violence may not be seeking healthcare in most cases. Population-level studies consistently indicate that the most common perpetrators of forced sex in both women and men are intimate partners $[17,26,27]$. The low number of survivors of intimate partner sexual violence seeking healthcare in Kenya is not surprising given the pervasive culture of acceptance of partner violence among women, stigma and a legal framework that does not recognise marital rape as a crime [28-31]. Nevertheless, the apparent high levels of survivors of intimate partner sexual violence not accessing healthcare is of immense concern when considered in light of health consequences, such as HIV transmission. A Kenya National AIDS Control Council report indicates that 
majority of new HIV infections occur among married or cohabiting couples [32]. Moreover, many of the HIV-infected couples are in discordant relationships where only one partner is infected [33, 34]. With forced sex, survivors in such relationships are at a heightened risk of HIV infection. Overall, evidence shows that IPV increases the risk of HIV acquisition among women by $50 \%$, not only through forced sex but also other indirect pathways [35]. Specific efforts to reach these survivors should explore alternative and culturally acceptable interventions that address the community norms while providing the necessary information and linkages to care. Notably, while IPV is likely to be a chronic problem and involve different forms of violence, the services currently available at the hospitals are set up to respond to acute cases of sexual violence and no guidelines exist on dealing with IPV.

\section{Gaps in healthcare services}

The HIV PEP initiation rate in our study (70\%) is consistent with previous studies that have documented rates of HIV PEP initiation ranging from 63 to $94 \%[15,19,20,24]$. However, the fact that $24 \%$ of survivors presented to the hospital within the recommended $72 \mathrm{~h}$ but were not put on HIV PEP is of great concern. The reasons for not being put on HIV PEP are unclear and require further investigation. Kenya is a country with high (5.6\%) HIV prevalence [34] and sexual violence represents a significant risk factor for HIV acquisition [36]. Hence, the need to start all survivors with potential exposure to HIV on prophylaxis is clear. It was notable that not being put on treatment for HIV PEP and other STIs was associated with certain survivor characteristics such as age, whether the perpetrator was known or unknown, and the perpetrator relationship to the survivor. While these findings may suggest that healthcare providers may be considering these characteristics when prescribing treatment, the national guidelines do not recommend using survivor characteristics as determinants for or against treatment. It is also possible that more treatments were administered than were documented. Not documenting treatment can compromise effective treatment as there are multiple departments involved. Additionally, not documenting other services rendered such as laboratory investigations may compromise treatment as well as effective follow-up. These findings therefore point to an area that needs to be urgently addressed in order to ensure that all survivors receive the recommended treatment.

Qualitative data indicates a need to streamline services within the hospital and for capacity building of HCPs.
Very few HCPs interviewed had received any form of training on sexual violence care. WHO recommends that all healthcare providers attending to survivors should receive training not only on the clinical management of survivors, but also on relevant legal and policy guidelines as well as ethical issues such as confidentiality and reporting requirements [5]. Data also showed incomplete documentation of clinical findings, laboratory investigations and treatment was common. Poor documentation is detrimental to both the clinical management of the survivor and for forensic evidence. Test results are especially important in making decisions on what treatment to give, for follow up of the survivor and some as legal evidence if the case goes to court. There is an urgent need of training more providers to ensure timely and quality care.

\section{Limitations in services for children and adolescents}

Children below 18 years constitute the vast majority of survivors being treated in the two hospitals, consistent with other studies done locally [15, 19-21, 24]. Although children constitute the highest proportion of those being treated, according to the VACS 2010 report, more than $90 \%$ of children who experience sexual violence do not access healthcare [18]. In addition, the current study shows that many of those children who presented did not get the necessary treatment because they presented much too late for some of the treatment to be effective. These findings are a major cause for concern given the potential long-term effects of sexual violence on children.

Children and adolescents constitute a majority of survivors being treated, however, there are no services tailored to children and HCPs lack training on managing children. While treatment for survivors is generally similar for adults and children, the dynamics of dealing with children and adolescents and their needs are vastly different from adults [37]. A recently published case study found that healthcare providers in Kenya lacked the basic skills necessary to deliver quality services to minors such as obtaining informed consent, maintaining confidentiality, conducting a physical examination that maintains the dignity of the child and collecting specimens [38]. Our qualitative interviews found that none of the healthcare providers in these facilities had received any training on dealing with children and adolescents. The national guidelines for treating survivors are highly adult-oriented with a few sections dedicated to the management of children. There is an urgent need to develop treatment protocols specific for children and adolescents, and to provide appropriate training to healthcare providers.

Protocols for treatment adolescents will need to address how to deal with young girls who are in 
consensual sexual relationships that are not socially and legally sanctioned as the girls have not reached the legal age of consent. This study indicated that more than 15\% of the survivors at the hospitals fell in this category where parents disapprove of these relationships and therefore take the girls to hospital for treatment as sexual abuse. While this is clearly a legal issue, healthcare providers are faced with an ethics and rights dilemma as these survivors might be brought to hospital against their will and subjected to examination and treatment that they do not wish to receive. It is not clear whether bringing these girls to hospital and treating them as sexual violence cases actually stops the on-going relationships. More importantly, there are no guidelines for clinicians on what rationale or criteria they should use to make decisions on the type of treatment and additional support to offer.

It is also noteworthy that while Kenyan children frequently report sexual violence occurring in schools in national surveys such as the VACS, only a few of the children presenting at the hospital reported this. Child sexual violence in schools perpetrated by educators, peers and other school staff is common globally [39-41]. It is not clear why these children were heavily under-represented among the hospital respondents. Nevertheless, this points to schools being an important area for outreach- not only for primary prevention of sexual violence but also to get children who experience violence in schools accessing healthcare. Thus, creating linkages between schools and healthcare resources is crucial.

\section{Limitations of the study}

Gaps in documentation were a main challenge for the quantitative hospital data. These gaps were inevitable, given our use of routinely collected clinical data. To overcome this challenge, multiple sources of data were used to fill in missing data. We also conducted the study at two hospitals. While these two hospitals are not representative nationally, they provide a good overview and are likely to capture a more wholesome picture of the survivors seeking treatment in Kenya than most previously-conducted research. As referral hospitals in two different counties, they attend to patients from both urban and rural settings, however, they are both located in urban centres. It is therefore likely that the profile of survivors attended to is different and sexual violence service provision differs (and is likely better) than more rural hospitals. Finally, the hospital data are not representative nationally.

The small number of HCPs interviewed limited qualitative data and therefore theoretical saturation may not have been reached. However, these HCPs were purposely chosen to represent those routinely attending to sexual violence survivors, capturing the most significant experiences and an in-depth knowledge of the issues involved.

The VACS data excludes children below 13 years and the KDHS excludes children below 15 years, therefore, no comparisons for children below 15 years were made. Additionally, while the KDHS survey asks about violence perpetrated by both intimate and non-intimate partners, information on intimate partner violence is collected in more details and the findings should be viewed with this in mind. Moreover, the study populations differed in terms of recall duration for the measures: in the surveys, we assessed survivors who had ever reported sexual violence while in the hospital data, survivors could present at any time, but it is more likely to have been recent sexual violence. Therefore, while younger people were more likely to present in the hospital data compared to older survivors in the KDHS data, this might reflect both age differences in reporting of sexual violence but may also be an indicator of how recent the violence was i.e. younger people having more recent violence and therefore presenting at the hospitals. Overall, triangulation of national survey data, quantitative and qualitative hospital data enabled our data to be more comprehensive.

\section{Conclusions}

Our study finds that multiple barriers both at the hospital and community level result in missed treatment opportunities for survivors. Although national guidelines are available, the operationalisation of these guidelines at the hospital level is still limited by lack of staff training, poor coordination between service delivery points, lack of specific protocols for different categories of survivors as well as unavailability of basic equipment such as HIV kits, speculums and rape kits. This not only hinders provision of quality healthcare to survivors but also the collection of forensic specimen necessary for legal procedures. Specific clinical guidelines and protocols for treating children and adolescents are needed. Additionally, as not documenting treatment differs significantly from not giving the treatment, the incomplete documentation in the hospital data is a serious problem that needs to be urgently addressed.

At the community level, older survivors; partnered or ever partnered survivors; survivors experiencing sexual violence from intimate partners; children experiencing violence in schools and men are at a higher risk of not accessing healthcare. Additionally, presenting late for treatment contributes to more survivors missing out on essential treatment. Interventions at the community level should reach out to those survivors who are unlikely to seek healthcare and address barriers to early access to care. 


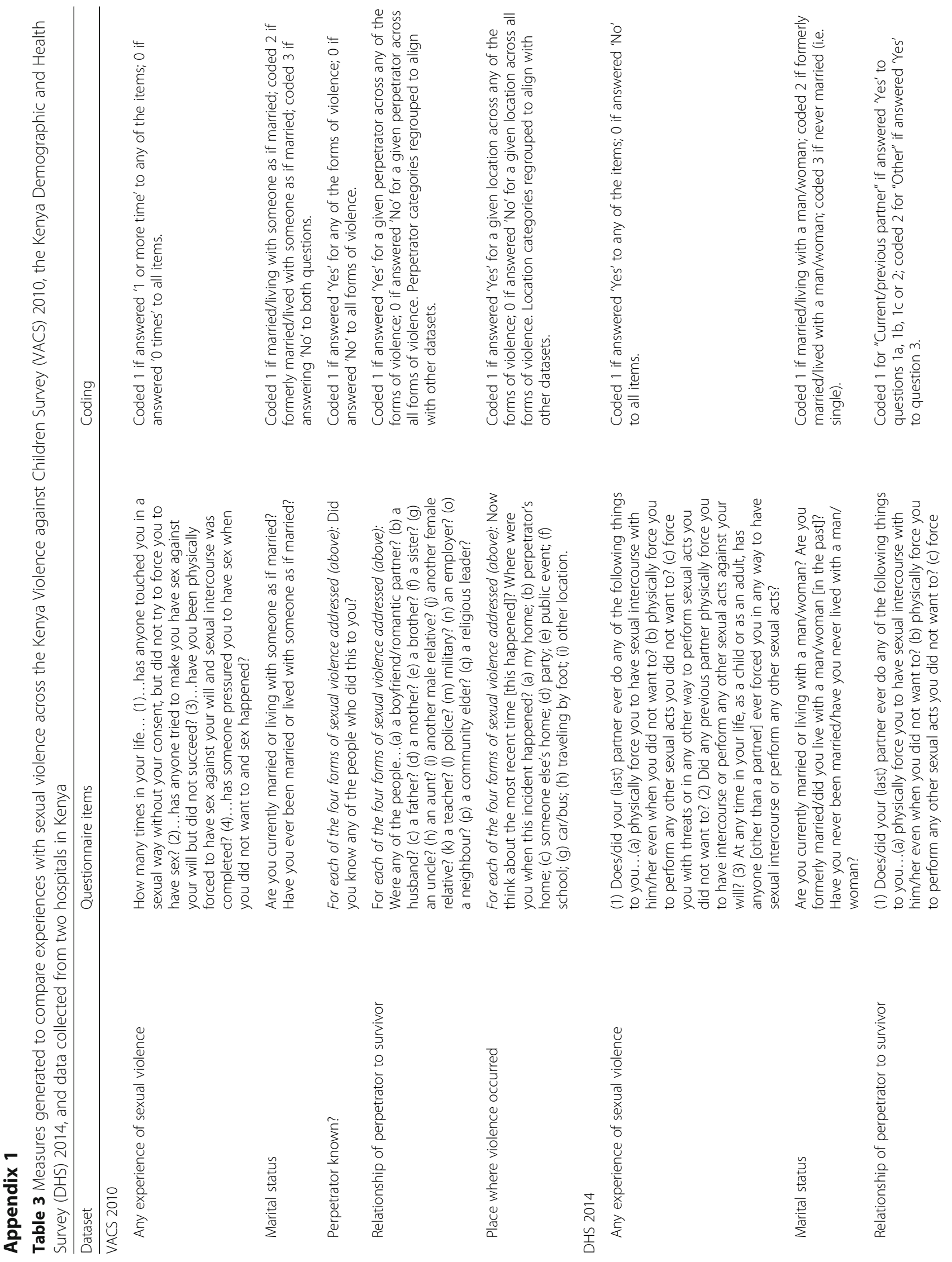


Gatuguta et al. BMC Public Health (2018) 18:769

Page 14 of 18
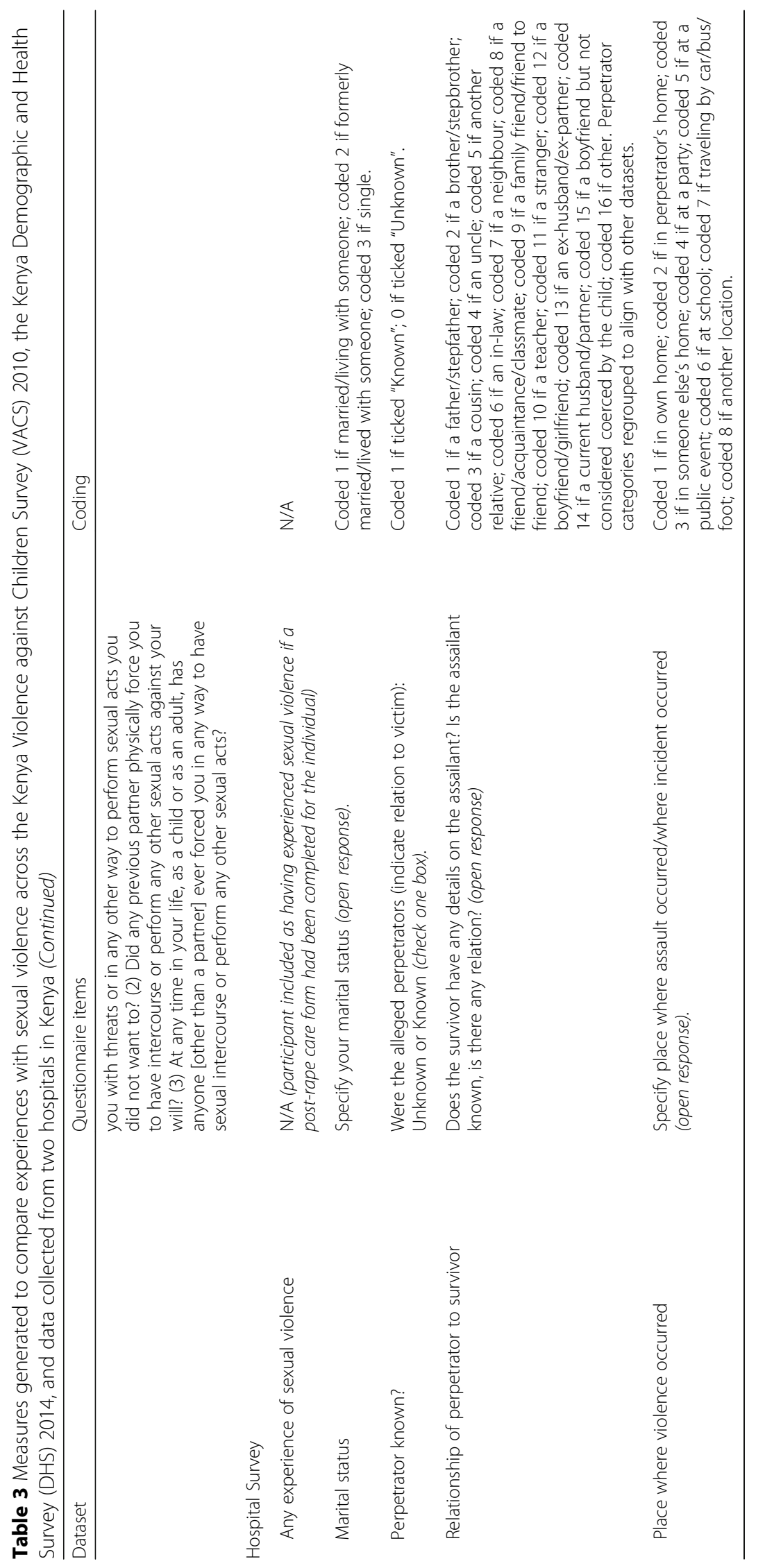


\section{Appendix 2}

Table 4 Socio-demographic characteristics of survivors seen at Naivasha and Thika Hospitals

\begin{tabular}{|c|c|c|c|c|c|}
\hline & & $\begin{array}{l}\text { Naivasha } \\
\text { Hospital n (\%) }\end{array}$ & $\begin{array}{l}\text { Thika Hospital } \\
\text { n (\%) }\end{array}$ & $\begin{array}{l}\text { Total } \\
\text { n (\%) }\end{array}$ & $P$ value $\left(X^{2}\right)$ \\
\hline \multirow[t]{3}{*}{ Gender } & Female & $313(93.4)$ & $193(92.6)$ & $506(93.2)$ & \multirow[t]{3}{*}{0.772} \\
\hline & Male & $22(6.6)$ & $15(7.4)$ & $37(6.8)$ & \\
\hline & Total & $335(100)$ & $208(100)$ & $543(100)$ & \\
\hline Age & Mean age in years (Range) & $16.4(2-67)$ & $17.6(1-51)$ & $16.8(1-67)$ & $\begin{array}{l}\text { t-test } P=0.1578 \\
\text { (Cl 15.990, 17.656) }\end{array}$ \\
\hline \multirow[t]{2}{*}{ Age group } & 18 years and below & $233(75.2)$ & $104(58.8)$ & $337(69.2)$ & \multirow[t]{2}{*}{$<0.001$} \\
\hline & Above 18 years & $77(24.8)$ & $73(41.2)$ & $150(30.8)$ & \\
\hline \multirow[t]{6}{*}{ Marital status } & Single & $54(61.4)$ & $69(77.5)$ & $123(69.5)$ & \multirow[t]{6}{*}{0.008} \\
\hline & Married & $21(23.9)$ & $11(12.4)$ & $32(18.1)$ & \\
\hline & Divorced & $3(3.4)$ & $4(4.5)$ & $7(3.9)$ & \\
\hline & Separated & $3(4.4)$ & $5(5.6)$ & $8(4.5)$ & \\
\hline & Widowed & $7(7.9)$ & 0 & $7(3.9)$ & \\
\hline & Total & $88(100)$ & $89(100)$ & $177(100)$ & \\
\hline \multirow{3}{*}{$\begin{array}{l}\text { Orphans \& vulnerable } \\
\text { children (OVC) }\end{array}$} & Yes & $14(4.4)$ & $13(9.1)$ & $27(5.9)$ & \multirow[t]{3}{*}{0.047} \\
\hline & No & $304(95.6)$ & $130(90.9)$ & $434(94.1)$ & \\
\hline & Total & $318(100)$ & $143(100)$ & $461(100)$ & \\
\hline \multirow[t]{3}{*}{ Disability } & Yes & $14(4.4)$ & $5(3.1)$ & $19(3.9)$ & \multirow[t]{3}{*}{0.485} \\
\hline & No & $308(95.7)$ & $159(97.0)$ & $467(96.1)$ & \\
\hline & Total & $322(100)$ & $164(100)$ & $486(100)$ & \\
\hline \multirow[t]{3}{*}{ Perpetrator known to survivor } & Known & $257(77.2)$ & $130(63.4)$ & $387(71.9)$ & \multirow[t]{3}{*}{0.001} \\
\hline & Unknown & $76(22.8)$ & 75 (36.6) & $151(28.1)$ & \\
\hline & Total & $333(100)$ & $205(100)$ & $538(100)$ & \\
\hline \multirow{7}{*}{$\begin{array}{l}\text { Perpetrator relationship } \\
\text { to survivor }\end{array}$} & Relative & $33(15.6)$ & $16(16.7)$ & $49(16.0)$ & \multirow[t]{7}{*}{0.017} \\
\hline & Neighbour & $66(31.3)$ & $28(29.2)$ & $94(30.6)$ & \\
\hline & Friend & $38(18.0)$ & 15 (15.6) & $53(17.3)$ & \\
\hline & Boyfriend (not coerced) & $40(19.0)$ & $7(7.3)$ & $47(15.3)$ & \\
\hline & Partner or ex-partner & $4(1.9)$ & $5(5.2)$ & $9(2.9)$ & \\
\hline & ${ }^{\mathrm{a}}$ Others & $30(14.2)$ & $25(26.0)$ & $55(17.9)$ & \\
\hline & Total & $211(100)$ & $96(100)$ & $307(100)$ & \\
\hline \multirow[t]{5}{*}{ Number of perpetrators } & 1 & $294(88.6)$ & $167(84.3)$ & $461(86.9)$ & \multirow[t]{5}{*}{0.053} \\
\hline & 2 & $28(8.4)$ & $19(9.6)$ & $47(8.9)$ & \\
\hline & 3 & $3(0.9)$ & $9(4.6)$ & $12(2.3)$ & \\
\hline & 4 or more & $7(2.1)$ & $3(1.5)$ & $10(1.9)$ & \\
\hline & Total & $332(100)$ & $198(100)$ & $530(100)$ & \\
\hline \multirow[t]{5}{*}{ Type of sexual violence } & Vaginal & $285(87.2)$ & $169(83.3)$ & $454(85.7)$ & \multirow[t]{5}{*}{0.207} \\
\hline & Anal & $23(7.0)$ & $17(8.4)$ & $40(7.6)$ & \\
\hline & Combined (Vaginal \& Anal/ Oral) & $5(1.5)$ & $9(4.4)$ & $14(2.6)$ & \\
\hline & Non-penetrative & $14(4.3)$ & $8(3.9)$ & $22(4.2)$ & \\
\hline & Total & $327(100)$ & $203(100)$ & $530(100)$ & \\
\hline \multirow[t]{4}{*}{ Place of sexual violence } & Perpetrator's home & $147(49.3)$ & $36(33.0)$ & $183(45.0)$ & \multirow[t]{4}{*}{0.001} \\
\hline & By the roadside/bush & 59 (19.8) & $24(22.0)$ & $83(20.4)$ & \\
\hline & Survivor's own home & $55(18.5)$ & $16(14.7)$ & $71(17.4)$ & \\
\hline & Someone else's home & $10(3.4)$ & $9(8.3)$ & $19(4.7)$ & \\
\hline
\end{tabular}


Table 4 Socio-demographic characteristics of survivors seen at Naivasha and Thika Hospitals (Continued)

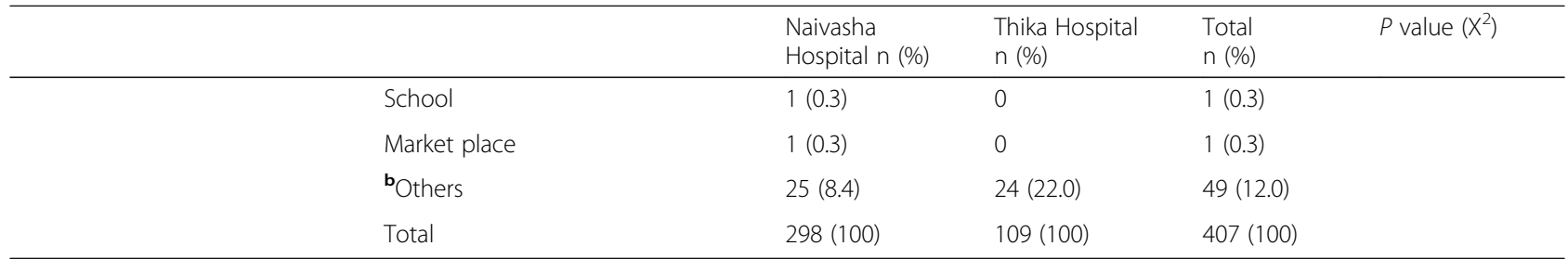

${ }^{a}$ Refers to perpetrators where only a few survivors reported each of them and include people known to the survivor through different associations such as local shopkeepers, employers and neighbourhood "motorcycle taxi" riders locally known as Boda bodas

${ }^{b}$ Other places where violence occurred include inside vehicles, abandoned buildings

\section{Appendix 3}

Table 5 Clinical examination and laboratory tests findings for survivors presenting at the two hospitals

\begin{tabular}{|c|c|c|c|c|c|}
\hline & & $\begin{array}{l}\text { Naivasha Hospital } \\
n=335 \mathrm{~N}(\%)\end{array}$ & $\begin{array}{l}\text { Thika Hospital } \\
n=208 \text { N (\%) }\end{array}$ & Total $n=543 n(\%)$ & $p$ value \\
\hline \multicolumn{6}{|l|}{ Clinical examination findings } \\
\hline \multirow[t]{3}{*}{ Physical exam } & Normal & $286(85.4)$ & $157(75.5)$ & $443(81.6)$ & \\
\hline & Abnormal & $42(12.5)$ & $29(13.9)$ & $71(13.1)$ & $<0.001$ \\
\hline & Not documented & $7(2.1)$ & $22(10.6)$ & $29(5.3)$ & \\
\hline \multirow[t]{5}{*}{ Genital exam } & Normal & $45(13.4)$ & $48(23.1)$ & $93(17.1)$ & \\
\hline & Hymen broken only & $173(51.6)$ & $79(38.0)$ & $252(46.4)$ & \\
\hline & Hymen broken with other signs & $91(27.2)$ & 49 (23.6) & $140(25.8)$ & 0.001 \\
\hline & Others e.g. anal signs & $21(6.3)$ & $25(12.0)$ & $46(8.5)$ & \\
\hline & Not documented & $5(1.5)$ & $7(3.4)$ & $12(2.2)$ & \\
\hline \multirow[t]{3}{*}{ Psychological assessment } & Calm/normal & $278(83)$ & $149(71.6)$ & $427(78.6)$ & \\
\hline & Not calm/ other psychological disturbance & $21(6.3)$ & $19(9.1)$ & $40(7.4)$ & 0.006 \\
\hline & Not documented & $36(10.7)$ & $40(19.2)$ & $76(14.0)$ & \\
\hline \multicolumn{6}{|l|}{ Laboratory test results } \\
\hline \multirow[t]{4}{*}{ HIV } & Negative & $296(88.4)$ & $25(12.0)$ & $321(59.1)$ & \\
\hline & Positive & $2(0.6)$ & 0 & $2(0.4)$ & $<0.001$ \\
\hline & Known HIV positive patient & $2(0.6)$ & $1(0.5)$ & $3(0.6)$ & \\
\hline & Not documented & $35(10.6)$ & $182(87.5)$ & $217(40.0)$ & \\
\hline \multirow[t]{3}{*}{ Urinalysis } & Normal & $206(61.5)$ & $20(9.6)$ & $226(41.6)$ & \\
\hline & Abnormal & $70(20.9)$ & $18(8.7)$ & $88(16.2)$ & $<0.001$ \\
\hline & Not documented & $59(17.6)$ & $170(81.7)$ & $229(42.2)$ & \\
\hline \multirow{3}{*}{$\begin{array}{l}\text { Swab microscopy (high vaginal, } \\
\text { oral or anal) }\end{array}$} & Normal & $108(32.2)$ & $29(13.9)$ & $137(25.2)$ & \\
\hline & Abnormal & $149(44.5)$ & $38(18.3)$ & $187(34.4)$ & $<0.001$ \\
\hline & Not documented & $78(23.3)$ & $141(67.8)$ & $219(40.3)$ & \\
\hline \multirow[t]{3}{*}{ Pregnancy test } & Positive & $30(12.8)$ & $3(7.5)$ & $33(12.0)$ & 0.339 \\
\hline & Negative & $204(87.2)$ & $37(92.5)$ & $241(88.0)$ & \\
\hline & Total & $234(100)$ & $40(100)$ & $274(100)$ & \\
\hline \multirow[t]{3}{*}{ VDRL } & Positive & $2(0.6)$ & 0 & $2(0.4)$ & \\
\hline & Negative & $296(88.4)$ & $10(4.8)$ & $306(56.4)$ & $<0.001$ \\
\hline & Not documented & $37(11.0)$ & $198(95.2)$ & $235(43.3)$ & \\
\hline \multirow[t]{3}{*}{ Hepatitis B surface antigen } & Positive & $1(0.3)$ & 0 & $1(0.2)$ & $<0.001$ \\
\hline & Negative & $268(80.0)$ & 0 & $268(49.4)$ & \\
\hline & Not documented & 66 (19.7) & $208(100)$ & $274(50.4)$ & \\
\hline
\end{tabular}




\section{Abbreviations}

CO: Clinical officer; EC: Emergency contraceptive; HCP: Health care provider; HIV: Human immunodeficiency virus; KDHS: Kenya demographic and health survey; PEP: Post exposure prophylaxis; PRC: Post rape care; SGBV: Sexual and gender based violence; STI: Sexually transmitted infection; VACS: Violence against children survey; VDRL: Venereal disease research laboratory; WHO: World Health Organisation

\section{Acknowledgements}

We are grateful to the staff at the two hospitals for their invaluable support during data collection. We are particularly grateful to the interviewees for their time and sharing their experiences. We also acknowledge the Demographic and Health Surveys (DHS) Program and the Centres for Disease Control and Prevention for access to the KDHS and VACs data respectively.

\section{Funding}

We acknowledge the funding by the Commonwealth Scholarship Commission for the PhD studentships for AG.

\section{Availability of data and materials}

The datasets generated and analysed during the current study are available from the corresponding author on request.

\section{Authors' contributions}

AG conceptualised and designed the study. KD, JS, MC, SS and IM contributed to the conceptualisation of the study. KG and AG analysed the data. AG drafted the manuscript and all authors critically reviewed drafts and approved the final manuscript.

\section{Ethics approval and consent to participate}

Ethical approval for the study was obtained from the institutional ethics review committee of London School of Hygiene and Tropical Medicine (Ref. 9896) and Kenyatta University (PKU/386/E32). All participants gave informed written consent.

\section{Consent for publication}

Not applicable

\section{Competing interests}

The authors declare that they have no competing interests.

\section{Publisher's Note}

Springer Nature remains neutral with regard to jurisdictional claims in published maps and institutional affiliations.

\section{Author details}

'Department of Global Health and Development, Faculty of Public Health and Policy, London School of Hygiene and Tropical Medicine, Keppel Street, London WC1E 7HT, UK. 'ㄹepartment of Community Health, School of Public Health, Kenyatta University, Nairobi, Kenya. ${ }^{3}$ Department of International Health, Johns Hopkins Bloomberg School of Public Health, Baltimore, MD, USA. ${ }^{4}$ Faculty of Epidemiology and Public Health, London School of Hygiene and Tropical Medicine, London, UK.

\section{Received: 4 July 2017 Accepted: 7 June 2018}

Published online: 19 June 2018

\section{References}

1. de Visser RO, et al. The impact of sexual coercion on psychological, physical, and sexual well-being in a representative sample of Australian women. Arch Sex Behav. 2007;36(5):676-86.

2. Etienne $K$, et al. World report on violence and health. Geneva: World Health Organization; 2002

3. McFarlane J, et al. Intimate partner sexual assault against women: frequency, health consequences, and treatment outcomes. Obstet Gynecol. 2005 105(1):99-108.

4. WHO. WHO multi-country study on women's health and domestic violence against women: summary report of initial results on prevalence, health outcomes and women's responses. Geneva: World Health Organization; 2005.
5. WHO. Guidelines for medico-legal care for victims of sexual violence. Geneva: World Health Organization; 2003.

6. Spataro J, et al. Impact of child sexual abuse on mental health: prospective study in males and females. Br J Psychiatry. 2004:184:416-21.

7. Ahrens KR, et al. Association between childhood sexual abuse and transactional sex in youth aging out of foster care. Child Abuse Negl. 2012; 36(1):75-80

8. Briere J, Elliott DM. Prevalence and psychological sequelae of self-reported childhood physical and sexual abuse in a general population sample of men and women. Child Abuse Negl. 2003;27(10):1205-22.

9. Lalor K, McElvaney R. Child sexual abuse, links to later sexual exploitation/ high-risk sexual behavior, and prevention/treatment programs. Trauma Violence Abuse. 2010:11:159-77.

10. Richter $\mathrm{L}$, et al. Reported physical and sexual abuse in childhood and adult HIV risk behaviour in three African countries: findings from project accept (HPTN-043). AIDS Behav. 2014;18(2):381-9.

11. de Visser RO, et al. Sex in Australia: experiences of sexual coercion among a representative sample of adults. Aust N Z J Public Health. 2003;27(2):198-203.

12. Abrahams N, Jewkes R. Barriers to post exposure prophylaxis (PEP) completion after rape: a south African qualitative study. Cult Health Sex. 2010;12(5):471-84

13. Chacko $L$, et al. Adherence to HIV post-exposure prophylaxis in victims of sexual assault: a systematic review and meta-analysis. Sex Transm Infect. 2012;88(5):335-41.

14. Linden $J A$, et al. HIV postexposure prophylaxis in sexual assault: current practice and patient adherence to treatment recommendations in a large urban teaching hospital. Acad Emerg Med. 2005;12(7):640-6.

15. Siika AM, et al. The structure and outcomes of a HIV Postexposure prophylaxis program in a high HIV prevalence setup in western Kenya. J Acquir Immune Defic Syndr. 2009;51(1):47-53. https://doi.org/10.1097/QAl. Ob013e318198a96a.

16. Institute for Health Metrics and Evaluation. Country profile: Kenya. Global Burden of Disease (GBD) 2013; Available from: http://www.healthdata.org/ kenya. Cited 8 Sep 2016

17. KNBS. Kenya Demographic and Health Survey 2014. Nairobi: Kenya National Bureau of Statistics; 2014

18. UNICEF. Violence against Children in Kenya: Findings from a 2010 National Survey. United Nations Children's Fund Kenya Country Office, Division of Violence Prevention, National Centre for Injury Prevention and Control, U.S. Centers for Disease Control and Prevention, Kenya National Bureau of Statistics: Nairobi, Kenya. 2012.

19. Speight $\mathrm{CG}$, et al. Piloting post-exposure prophylaxis in Kenya raises specific concerns for the management of childhood rape. Trans R Soc Trop Med Hyg. 2006;100(1):14-8.

20. Ranney ML, et al. A novel ED-based sexual assault centre in western Kenya: description of patients and analysis of treatment patterns. Emerg Med J. 2011:28(11):927-31.

21. Kilonzo N, et al. Delivering post-rape care services: Kenya's experience in developing integrated services. Bull World Health Organ. 2009:87(7):555-9.

22. $\mathrm{MOH}$. National guidelines on management of sexual violence in Kenya. Nairobi: Ministry of Health; 2014

23. Pope C, Ziebland S, Mays N. Qualitative research in health care: analysing qualitative data. BMJ. 2000:320:114-6

24. Buard V, et al. Characteristics, medical management and outcomes of survivors of sexual gender-based violence, Nairobi, Kenya. Public Health Action. 2013;3(2):109-12.

25. Christian M, et al. Sexual and gender based violence against men in the Democratic Republic of Congo: effects on survivors, their families and the community. Med Confl Surviv. 2011;27(4):227-46.

26. KNBS. Kenya Demographic and Health Survey 2008-09. Calverton: Kenya National Bureau of Statistics (KNBS) and ICF Macro; 2010.

27. Adudans MK, et al. Prevalence of Forced Sex and Associated Factors among Women and Men in Kisumu, Kenya. Afr J Reprod Health. 2011;15(4):87-97.

28. Winfred Kamau. Legal treatment of consent in sexual offences in Kenya. 2013; Available from: http://theequalityeffect.org/pdfs/ConsentPaperKenya. pdf. Cited 18 Mar 2015

29. Hatcher AM, et al. Social context and drivers of intimate partner violence in rural Kenya: implications for the health of pregnant women. Cult Health Sex. 2013;15(4):404-19

30. The National Council for Law Reporting. The Sexual Offences Act, No.3 of 2006. Nairobi: The National Council for Law Reporting; 2006. 
31. Odero $\mathrm{M}$, et al. Responses to and resources for intimate partner violence: qualitative findings from women, men, and service providers in rural Kenya. J Interpers Violence. 2014;29(5):783-805.

32. NACC. HIV prevention response and modes of transmission analysis. Nairobi: Kenya National AIDS Control Council; 2009.

33. Kaiser $\mathrm{R}$, et al. Factors associated with HIV infection in married or cohabitating couples in Kenya: results from a nationally representative study. PLoS One. 2011;6(3):e17842.

34. NASCOP. Kenya AIDS Indicator Survey 2012: Final Report. Nairobi: National AIDS and STI Control Programme; 2014.

35. Heise L, McGrory E, Greentree II. Violence against Women and Girls, and HIV. London: London School of Hygiene \& Tropical Medicine; 2016.

36. WHO. Post-exposure prophylaxis to prevent HIV infection : joint WHO/ILO guidelines on post-exposure prophylaxis (PEP) to prevent HIV infection. 2007; Available from: http://www.who.int/hiv/pub/guidelines/PEP/en/. Cited 20 Feb 2016.

37. Population Council. Sexual and gender based violence in Africa: literature review. Nairobi: Population Council; 2008.

38. Wangamati $\mathrm{CK}$, et al. Postrape care services to minors in Kenya: are the services healing or hurting survivors? Int J Women's Health. 2016;8:249-59.

39. UNICEF, Violence against children in schools and education settings. New York: United Nations International Children's Emergency Fund. 2006. p. 109169.

40. Devries KM, et al. School violence, mental health, and educational performance in Uganda. Pediatrics. 2014;133(1):e129-37.

41. Prinsloo S. Sexual harassment and violence in South African schools. South African J Educ. 2006;26(2):305-8.

Ready to submit your research? Choose BMC and benefit from:

- fast, convenient online submission

- thorough peer review by experienced researchers in your field

- rapid publication on acceptance

- support for research data, including large and complex data types

- gold Open Access which fosters wider collaboration and increased citations

- maximum visibility for your research: over $100 \mathrm{M}$ website views per year

At BMC, research is always in progress.

Learn more biomedcentral.com/submissions 